\title{
Mode II crack paths under compression in brittle solids - a theory and experimental comparison
}

\author{
P. Isaksson *, P. Ståhle \\ Division of Solid Mechanics, Malmö University, SE-205 06 Malmö, Sweden \\ Received 15 March 2001; received in revised form 24 November 2001
}

\begin{abstract}
A study of crack propagation paths in the situation where the crack is suppressed to open during growth due to high compressive forces has been performed. This problem was analyzed theoretically very recently by the authors and is here extended to involve a limited number of illustrative experimental results reported elsewhere in the literature. By analyzing the experimental crack growth patterns, the conclusion is that the model cannot describe the more realistic microscopic failure in detail. Since shear crack growth on the microscale strongly depend on inhomogenities in the material, like cavities, grains or inclusions; the closed crack growth patterns observed are not smooth or free of kinks. Nevertheless, the model show good agreement with the reported experimental observations of the paths of closed macroscopic mode II cracks on samples in brittle materials, induced under overall compression.

Failure patterns experimentally observed supports the theory that the growth of mode II cracks under compression in brittle materials follow a propagation path described by a function $y=\lambda x^{b}$, where $b=3 / 2$. This is strongly supported by the measured values obtained from various experiments. In all the studied experiments, the exponent $b$ was found in the interval [1.43-1.58]. Further, an investigation of the curvature parameter $\lambda$ has been performed and the conclusion is that $\lambda$ does also agree with the simplified model, even though not as good as the exponent $b$. However, $\lambda$ differs in general $<15 \%$ from the theoretical value predicted by the model. The process of crack growth is in the simplified model assumed to be controlled by the mode II stress intensity factor $K_{\mathrm{II}}$ of the main crack and the difference between the compressive remote normal stress parallel with the crack plane $\left(\sigma_{11}^{\infty}\right)$ and the compressive remote normal stress perpendicular to the crack plane $\left(\sigma_{22}^{\infty}\right)$. (c) 2002 Elsevier Science Ltd. All rights reserved.
\end{abstract}

Keywords: Crack growth; Mode II; Brittle material; Compressive stresses; Frictionless contact

\section{Introduction}

Cracks in brittle materials are often governed by linear elastic fracture mechanics. To this end crack initiation and propagation have been the subjects of intensive investigation in rock- and fracture mechanics, both experimentally and theoretically. However, most of the previous studies are focused on the

\footnotetext{
${ }^{*}$ Corresponding author.

E-mail address: peri@ts.mah.se (P. Isaksson).
} 
mechanisms of crack initiation and propagation of opening (mode I) cracks (cf. Erdogan and Sih, 1963; Bilby and Eshelby, 1968; Cotterell and Rice, 1980; or Hayashi and Nemat-Nasser, 1981a, 1981b). Experimental observations indicate that pure mode I crack growth is usually preferred before crack growth under mixed-mode or pure shear mode (mode II) conditions. Even under pure shear loading, the crack generally grows under mode I conditions as a result of local tensile stresses at the tip. For opening mode, an often used criterion is assumed crack extension in the plane of maximum tangential stress. Another frequently used propagation criterion, also for opening mode, is the assumption of crack growth through an incipient kink in a direction of vanishing mode II stress intensity factor $K_{\mathrm{II}}$. However, according to results reported by Melin $(1986,1987)$, can mode II crack growth in a brittle material be preferred if a high confining pressure is present or the when ratio between the critical stress intensity factors, $K_{\text {IIc }} / K_{\text {Ic }}$, is surprisingly low.

There are several interpretations of mode II failure, and the most common view is that shear mode crack growth is caused by a linkage of many small tensile cracks, so-called microcracks, (cf. Broberg, 1987; Shen, 1993; or Li, 1999). Broberg (1987) argued that on a microscale, mode II growth could consist of repeated microseparations at an angle to the crack direction due to tensile forces of short reach near the crack tip. The zigzag crack paths often seen in mode II growth indicate this shear mode microseparation. In other cases the microseparation mechanism could consist of pure shear flow resulting in pure sliding of the crack surfaces. However, these mixed microseparation mechanisms occur as a result of the local conditions near the crack tip. It is believed that at a mixed mode I + II fracture, the crack grows in a direction that maximizes one of the stress intensity factors $K_{\mathrm{I}}$ or $K_{\mathrm{II}}$, rather than in a direction that maximizes some functional of $K_{\mathrm{I}}$ and $K_{\mathrm{II}}$. Furthermore, Broberg (1987) argues that if the compressive stress field at the crack tip is so high that an opening crack cannot propagate more than a very short distance from the tip because compressive stresses force the separation to close at its end, the continued crack growth possibly occur in the direction of the main crack, independent of the processes taking place in the microscale. A high confining pressure will then prohibit crack opening of the growing crack regardless the direction of crack growth. Compressive load may, therefore, create a dense array of microcracks. The crack will then grow by successive coalescence with microcracks in a narrow band extending from the crack edge, Broberg (1999). A more detailed discussion of the nucleation mechanism of microcracks in brittle materials can be found in, among others, (Broberg, 1987; Suresh, 1994; or Bobet and Einstein, 1998).

Thus, at a sufficiently high confining pressure leading to a vanishing opening stress intensity factor $K_{\mathrm{I}}=0$ at the tip of a kink in any direction, the crack is assumed to extend along a smooth curved path that maximizes the mode II stress intensity factor $K_{\text {II }}$ on the macroscale. One arrives at the same conclusion assuming crack growth along the plane of maximum shear stress as by assuming that the crack follows the direction of the largest $K_{\mathrm{II}}$.

Heterogeneous brittle materials, such as rock, concrete and ceramic, contain a large number of different inhomogenities (e.g. soft and hard inclusions such as voids, pores and microcracks). These defects provide the crack tip with a rather large process region, which may be responsible for the macroscopic mode II failure under compressive loads in such materials. In order to figure out conditions favoring mode II fracture Jung et al. (1992) conducted extended tests of pure shear loading on various brittle materials. It was reported that mode II crack growth occurred under pure shear loading in some materials, such as rock, while mode I crack growth occurred in other materials like glass or PMMA. A similar study is found in Melin (1989) where fracture paths in concrete, mortar and PMMA were analyzed and compared. Some researchers have reported that experiments conducted in brittle materials can result in propagation of a mode II crack when the compressive load is substantially high, following the occurrence of a mode I crack (cf. Lajtai, 1974; or Petit and Barquins, 1988). More recently (e.g. Reyes, 1991; Reyes and Einstein, 1991; Shen, 1993; Shen et al., 1995; Bobet and Einstein, 1998; Rao, 1999; or Bobet, 2000) pure mode II cracks were obtained in experiments conducted in brittle materials under uniaxial or biaxial compressive load. As confinement increased cracking was only produced by mode II cracks as observed by Bobet (2000), who in 
experiments and in a stress analysis concluded that shear cracks always initiated in compressive stress fields and propagated as mode II cracks on the macroscale. Thus, the result reported by Bobet (2000) verifies the theoretical results discussed by Melin (1986) in an analysis of crack kinking under confining stresses. Hence, there is a large body of evidence showing that mode II cracks may appear under compression, at least on a macroscale.

Recently Leblond and Frelat (2000) analyzed crack deflection from an initially closed stationary crack in a linearly elastic material. Although they assume the crack to be initially closed at the onset of crack growth due to compressive forces, the continued crack growth is supposed to occur under mode I conditions after initial kinking, which can be abrupt. Consequently, their crack path criterion is only applicable when the crack is allowed to open up. However, currently the authors analyzed (Isaksson and Ståhle, 2001), theoretically, the macroscale mode II crack propagation path in a brittle material under influence of high compressive normal stresses. An expression for the mode II stress intensity factor at a crack extension was found under the condition that the crack opening is suppressed during crack growth. The expression was found as a function of the mode II stress intensity factor $K_{\mathrm{II}}$ at the parent crack and the difference between the compressive remote normal stress $\sigma_{y}^{\infty}$ perpendicular to, and the normal stress $\sigma_{x}^{\infty}$ parallel with, the parent crack plane. Based on this expression, a crack growth direction model was suggested. At a sufficiently high non-isotropic compressive normal stress, so that the crack remains closed, the model predicts that the crack will extend along a path that maximizes the mode II stress intensity factor. The mode II crack growth most likely follows a curved path and the deflection from the original crack plane increases with increasing non-isotropic biaxial compressive normal stress $\sigma_{x}^{\infty}-\sigma_{y}^{\infty}$. Only at isotropic remote compressive normal stress the crack will continue straight ahead without change of the direction.

To verify the applicability of the model to real problems in rock mechanics, a comparison with experimental data is necessary. The objective of this paper is to investigate the curvature of growing mode II cracks where the initiation and propagation of the crack is controlled by a shear stress. The crack contours are obtained from various experiments reported on brittle materials in the literature.

\section{The problem}

Cracks subjected to shear load under high remote compressive normal stresses in brittle isotropic materials are considered. Numerous experimental and theoretical efforts have been devoted to the understanding of the crack initiation and propagation of pre-existing cracks in brittle materials, even though the attention mainly has been focused on mode I crack growth. Increasing attention has been paid to mode II crack growth in rock, but there is still a lack of systematic research such have been done for mode I failure. Not only are the classical mixed mode fracture criteria incapable of predicting the mode II crack growth under compressive load, but also it seem as the process of shear crack growth is not well understood. Samples in various brittle materials (like marble or gypsum) have demonstrably cracked in shear mode in experiments conducted under compressive loads (cf. Reyes, 1991; Reyes and Einstein, 1991; Shen, 1993; Shen et al., 1995; Bobet and Einstein, 1998; Rao, 1999; or Bobet, 2000). A review of the experiment setups is found in the Appendix A. In the reported experiments the authors argued that there is clear evidence that mode II cracks do initiate and propagate. After examination of the cracks it was found that some of the developed cracks were mode II cracks or a combination of a mode I crack followed by a mode II crack growth. The mode II cracks initiated from tips of pre-existing cracks and were reported to propagate in a stable manner. It is not sure if the initiated cracks were mode II cracks on a microscale (i.e. grain scale) but, as Bobet and Einstein (1998) concluded, on a macroscale the cracks were indeed mode II cracks. Later, in a stress analysis by Bobet (2000), it was concluded that the direction of the mode II cracks were determined by the direction of the maximum shear stress and the cracks were initiated (and propagated) in a compressive stress field. 


\section{Analysis of the mode II crack propagation path}

In all experimental configurations the mode II crack surfaces, during crack propagation, were subjected to a remote biaxial compressive stress field consisting of three main components; a compressive normal stress perpendicular to the crack plane $\left(\sigma_{22}^{\infty}\right)$, a compressive normal stress parallel with the crack plane $\left(\sigma_{11}^{\infty}\right)$ and a shear stress parallel to the crack plane $\left(\sigma_{12}^{\infty}\right)$. The first two stress components lead to the closing of the crack surfaces while the shear stress acts as the driving force for the continued crack growth, which is predominantly mode II in character.

The resulting crack growth patterns emerging from pre-existing cracks are analyzed. Since the preexisting cracks in the experiments considered are straight and subjected to compression, the shear crack propagation path model recently discussed by the authors elsewhere (Isaksson and Ståhle, 2001) is believed to be appropriate when analyzing the subsequent crack growth of a mode II crack under compressive load. In reality, most of the pre-existing cracks in brittle materials will close when subjected to a compressive stress, and most of the cracking will therefore effectively occur from closed cracks. The fracture process is assumed to occur under mode II conditions since a high confining pressure suppresses the crack opening. Therefore the crack is believed to extend along a path that maximizes the mode II stress intensity factor $K_{\text {II }}$, as discussed above. This assumption is equivalent with a criterion for maximum energy release rate as crack opening is suppressed. One arrives at the same conclusion assuming crack growth along the plane of maximum shear stress as by assuming that the crack follows the direction of the largest $K_{\mathrm{II}}$.

In Isaksson and Ståhle (2001), an expression for the mode II stress intensity factor at the tip of a kink was calculated, both numerically with an integral equation using the solution scheme found by Lo (1978) and later refined by $\mathrm{He}$ and Hutchinson (1989), and by particular analytic solutions. By distributing dislocations along a straight kink contour such that the stress boundary conditions across and along the kink was fulfilled (i.e. kink opening was prohibited), an expression describing the curved closed mode II crack extension in front of a pre-existing straight stationary crack could be found. The confidence in the solution was strengthened by analyzing the same problem using a boundary element method. Here follows a brief summary of the crack path prediction and the interested reader is advised to the original article for a more comprehended discussion.

Consider a locally plane structure initially containing a sharp semi-infinite crack, Fig. 1. A Cartesian coordinate system $\left(x_{1}, x_{2}, x_{3}\right)$, and a polar coordinate system $\left(r=\left(x_{1}^{2}+x_{2}^{2}\right)^{1 / 2}, \theta=\tan ^{-1}\left[x_{2} / x_{1}\right], z=x_{3}\right)$ are attached to the crack tip. The crack occupies the region $x_{1} \leqslant 0$ and $x_{2}=0$. The extent of the body in the $x_{3}-$ direction is assumed large wherefore plane strain conditions are assumed to prevail. The body is subjected to a high remote biaxial compressive stress, not necessarily isotropic, and loaded so that shear stresses are
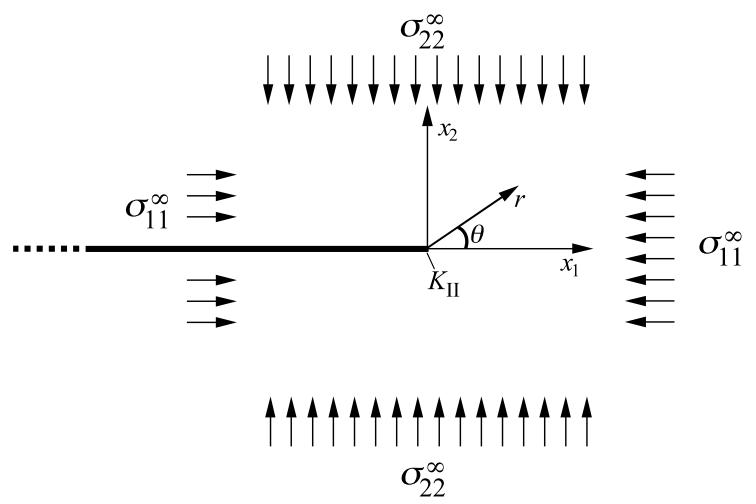

Fig. 1. Geometry and load of the crack. 


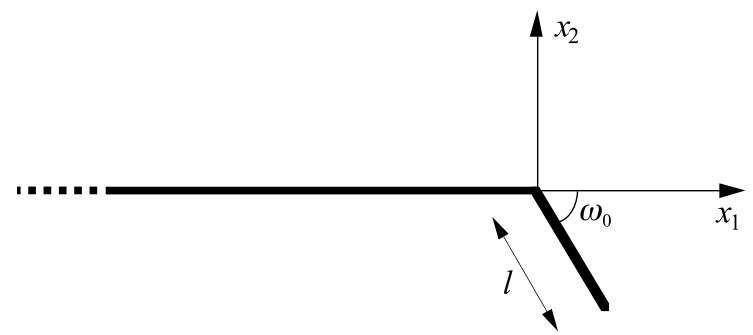

Fig. 2. Quantities of the kinked crack. The kink, of length $l$, is extended in an angle $\omega_{0}$.

present in the crack plane wherefore a mode II stress intensity factor $K_{\text {II }}$ has arised at the crack tip. Moreover, suppose the compressive load is sufficiently high leading to a vanishing opening mode I stress intensity factor $\left(K_{\mathrm{I}}=0\right)$. Assuming homogenous material and linearly elastic isotropic behavior, the first two terms of the stress tensor at the crack tip may be expressed as

$$
\sigma_{i j}=(2 \pi r)^{-1 / 2} K_{\mathrm{II}} f_{i j}^{\mathrm{II}}(\theta)+\sigma_{i j}^{\infty} \delta_{i j} \text { as } r \rightarrow 0 \quad \text { for } i=1,2 \text { and } j=1,2 .
$$

The dimensionless angular function $f_{i j}^{\mathrm{II}}(\theta)$ can be found in any textbook of fracture mechanics. Eq. (1) yields as long as the mode I component of the stress intensity factor is vanishing.

Introduce the non-isotropic stress term $\sigma_{11}^{\infty}-\sigma_{22}^{\infty}$, i.e. the difference between the remote compressive normal stress parallel with, and the remote compressive normal stress perpendicular to, the crack plane. Let the stress difference term $\sigma_{11}^{\infty}-\sigma_{22}^{\infty}$ be regarded as a superimposed uniaxial stress in the $x_{1}$-direction on a homogenous stress $\sigma_{22}$. Eq. (1) can be rewritten as

$$
\sigma_{i j}=(2 \pi r)^{-1 / 2} K_{\mathrm{II}} f_{i j}^{\mathrm{II}}(\theta)+\left(\sigma_{11}^{\infty}-\sigma_{22}^{\infty}\right) \delta_{1 i} \delta_{1 j}+\sigma_{22}^{\infty} \delta_{i j} .
$$

The stress state of the remote biaxial normal compressive stress is pure isotropic when $\sigma_{11}^{\infty}-\sigma_{22}^{\infty}=0$.

The crack is assumed to form a single incipient straight kink in a small angle $\omega_{0}$ to the crack plane as illustrated in Fig. 2. The angle $\omega_{0}$ is with respect to a clockwise rotation from the crack plane. The kink length $l$ is understood to be small, compared to the length of the parent crack, wherefore the asymptotic problem for a semi-infinite stationary parent crack is analyzed. The key result presented is the expression

$$
\omega_{0}=\frac{8}{13} \sqrt{\frac{2 l}{\pi}} \frac{\sigma_{11}^{\infty}-\sigma_{22}^{\infty}}{K_{\mathrm{II}}}
$$

where $\omega_{0}$ is the angle from the previous crack plane to the new assumed direction of crack growth. It was shown that the (small) angle $\omega_{0}$ gives a good approximation of the deflection angle for the straight kink that maximizes the mode II stress intensity factor $k_{2}$ at the kink tip for small values of load ratio $\left(\sigma_{11}^{\infty}-\sigma_{22}^{\infty}\right) l^{1 / 2} / K_{\mathrm{II}}$. The sign of $K_{\mathrm{II}}$ is with reference to a local Cartesian coordinate system at the crack tip with an axis $\left(x_{1}\right)$ in the direction of the extension of the crack plane before deflection and the other axis perpendicular to this in the counter clock-wise direction $\left(x_{2}\right)$. The continued crack growth is assumed to follow a smoothly curved path for which the shear stress intensity factor $k_{2}$ at the branched tip is maximized at all times.

From Eq. (3) it follows that when the remote biaxial compressive normal stress is isotropic (i.e. $\left.\sigma_{11}^{\infty}=\sigma_{22}^{\infty}\right)$ the crack will continue straight ahead without change of direction. The superimposed isotropic compressive stress $\sigma_{11}^{\infty}=\sigma_{22}^{\infty}$ is believed to have no significant importance for the kinking problem, except to close the crack surfaces, and can therefore be neglected if friction between the crack surfaces is disregarded. The effect of friction is discussed later. 

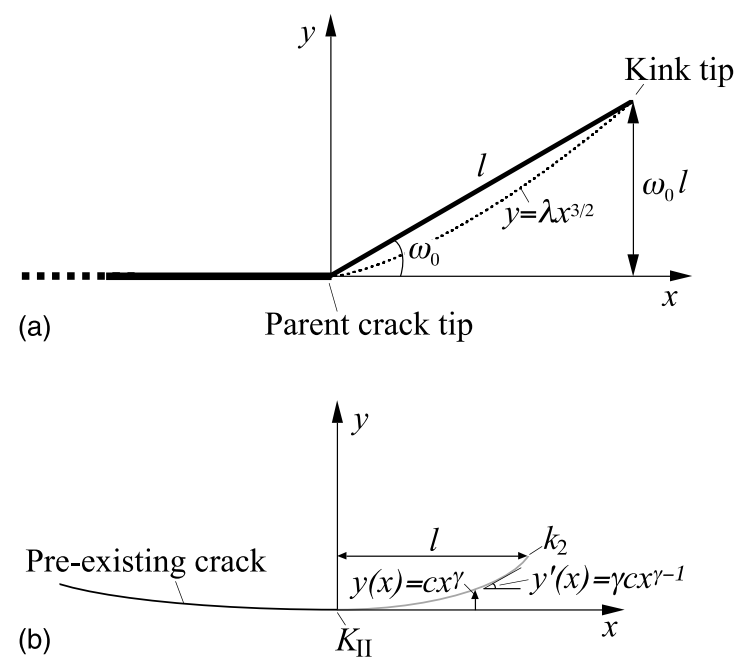

Fig. 3. (a) Deflection of the kink. (b) Small extension of a closed fracture from a pre-existing crack.

For small $\omega_{0}$, i.e. $y \ll x$, the deflection at the end of the kink is $y=\omega_{0} l$ as shown in Fig. 3a,

$$
y=\omega_{0} l=\lambda l^{3 / 2},
$$

where

$$
\lambda=\frac{8}{13} \sqrt{\frac{2}{\pi}} \frac{\sigma_{11}^{\infty}-\sigma_{22}^{\infty}}{K_{\mathrm{II}}} .
$$

The radius of curvature, $R$, is for small $\mathrm{d} y / \mathrm{d} x$ given by

$$
\frac{1}{R}=\frac{\mathrm{d}^{2} y}{\mathrm{~d} x^{2}}=\frac{3}{4} \lambda l^{-1 / 2}
$$

By putting $l \approx x$ in Eq. (4)

$$
y=\lambda x^{3 / 2} \text {, }
$$

which is similar to the expression found for the curvature of opening cracks discussed by Leblond and Frelat (2000) in a study of crack extension of an initially closed crack.

Thus, in theory, the crack is hypothesized to extend along a smooth curved path described by Eq. (6). Since the deflection of the kink from the parent crack plane is assumed to be small, Eq. (3) is believed to give an appropriate crack path prediction on the macroscale for a straight kink extension as a function of $K_{\text {II }}$, the remote normal stress difference term $\sigma_{11}^{\infty}-\sigma_{22}^{\infty}$ and the kink length $l$.

Eq. (6) may also be justified by simply considering the stress field at a stationary pure mode II crack tip. For small angles $\theta$ the angular functions $f_{i j}^{\mathrm{II}}(\theta)$ in Eq. (1) may be approximated by linear functions of $\theta$ since $\sin \theta \approx \theta$ and $\cos \theta \approx 1$ in a first order approximation. Consequently, as $y \ll x, \theta=\tan ^{-1} y / x \approx y / x$, and $r=\left(x^{2}+y^{2}\right)^{1 / 2} \approx x$, the stress field in front of the mode II crack tip can be approximated to:

$$
\begin{aligned}
& \sigma_{11}-\sigma_{11}^{\infty} \approx-\frac{K_{\mathrm{II}}}{\sqrt{2 \pi}} x^{-1 / 2} \frac{y}{x}=a x^{-3 / 2} y+O(y / x)^{2}, \\
& \sigma_{22}-\sigma_{22}^{\infty} \approx \frac{K_{\mathrm{II}}}{2 \sqrt{2 \pi}} x^{-1 / 2} \frac{y}{x}=b x^{-3 / 2} y+O(y / x)^{2},
\end{aligned}
$$


and

$$
\sigma_{12} \approx \frac{K_{\mathrm{II}}}{\sqrt{2 \pi}} x^{-1 / 2}+O(y / x)^{2},
$$

where $a$ and $b$ are arbitrary constants. In order to fulfill the boundary conditions that the normal stresses $\sigma_{11}-\sigma_{11}^{\infty}$ and $\sigma_{22}-\sigma_{22}^{\infty}$ have to remain constant (independent of small $\theta$ ) in front of the crack tip, one realizes that $y=c x^{3 / 2}$ is the only possible non-trivial solution to Eqs. (7) and (8). An arbitrary constant $c$ describes the curvature of the path. The conclusion is that an equation describing the curved closed mode II crack propagation path in front of a pre-existing straight stationary crack is required to be described by a function $y=c x^{3 / 2}$ in order to maintain the stress boundary conditions.

As the continued mode II crack growth is assumed to extend along a smooth curved path, one realizes that an extension of a straight kink not fully can describe the crack propagation path since the stress intensity factors of a straight and a curved crack extension are expected to differ. By assuming that the crack deflection from the $x$-axis can be described by a function $y=c x^{\gamma}$ (see Fig. 3b) calculations of the mode II stress intensity factor at the tip of a straight extension $(\gamma=1)$ and at the tip of a curved extension $(\gamma=3 / 2)$ were performed analytically in Isaksson and Ståhle (2001) using a solution method introduced by Cotterell and Rice (1980). An assumption of small deflection $y$ (i.e. $y \ll x$ ) was made for the crack extension along the $x$-axis with the parent crack tip located at the origin, Fig. $3 \mathrm{~b}$. The deflection angle of the curved extension along the $x$-axis was given by $\theta=y^{\prime}(x)$, where $y^{\prime}(x)=\partial y(x) / \partial x$. It was shown that the asymptotic value of the curvature parameter $c$ maximizing the mode II stress intensity factor $k_{2}$ at the tip of a crack extension described by the function $y=c x^{\gamma}$ is

$$
c=-\frac{8}{13} \sqrt{\frac{2}{\pi}} \frac{\left[\sigma_{11}^{\infty}-\sigma_{22}^{\infty}\right] \sqrt{l}}{K_{\mathrm{II}}} \approx-0.491 \frac{\left[\sigma_{11}^{\infty}-\sigma_{22}^{\infty}\right] \sqrt{l}}{K_{\mathrm{II}}} \quad \text { for } \gamma=1,
$$

and

$$
c=-\frac{8 \sqrt{2 \pi}}{39} \frac{\left[\sigma_{11}^{\infty}-\sigma_{22}^{\infty}\right]}{K_{\mathrm{II}}} \approx-0.514 \frac{\left[\sigma_{11}^{\infty}-\sigma_{22}^{\infty}\right]}{K_{\mathrm{II}}} \text { for } \gamma=3 / 2
$$

Thus, it seems justified to conclude that the straight kink model can, on the macroscale, find an approximate angle that maximizes the stress intensity factor $k_{2}$ for a curved extension.

In order for the analytical model to predict a crack propagation path with a curvature that is independent of the extension length $l$, the exponent $\gamma$ in the expression $y=c x^{\gamma}$ describing the crack propagation path is required to be equal to 3/2, Isaksson and Ståhle (2001). In that case, when $\gamma=3 / 2$, is the expression predicting the crack propagation path a function only of the mode II stress intensity factor $K_{\mathrm{II}}$ at the parent crack and the difference between the compressive normal stress parallel with $\left(\sigma_{11}^{\infty}\right)$, and perpendicular to $\left(\sigma_{22}^{\infty}\right)$, the parent crack plane.

\subsection{General assumptions and limitations}

As this simplified model does not consider friction effects, nor de-cohesion effects, the influence of the shielding effect due to arised friction on the curved crack extension is not considered. One realizes that friction along the crack surfaces may have an influence on the crack growth under our circumstances since the crack surfaces are pressed together. Shen (1993) reported that friction has a great influence on the fracture toughness ratio $K_{\text {Ic }} / K_{\text {IIc }}$. Since a possible mode II growth will occur straight ahead, or in small angle to, the previous crack plane it is believed that the friction has a larger affect on the mode of the growth 
rather than the direction of the continued mode II crack growth, as discussed theoretically by Melin (1986) in an analysis of crack kinking in a linear-elastic material under high confining stresses. A thorough analysis that takes full attention to the effect of friction on the curvature is regarded to be cumbersome and comprehends many difficulties. Nevertheless, one may draw some conclusions since the trend in the initially mode II crack propagation path should be the same. The argument that justifies this is as follows: at a sufficiently large compressive load, as compared to remote load, the crack cannot extend via a kink under opening mode I conditions. The only possibility seems to be mode II crack growth that occurs in a direction straight ahead of the crack plane if the crack is supposed to follow the plane of maximum shear stress. Thus the continued crack growth is assumed to follow a (on a macroscale) smoothly curved path co-planar, or in a small angle, to the original crack plane. The in-plane shear stress field is affected when friction is present between the crack surfaces. As $K_{\mathrm{II}}$ is controlled by the in-plane shear stress, Eqs. (3) and (11) are to some extent affected by friction, and in turn, the supposed deflection of the crack extension according to the model.

The conclusion is that the proposed model is restricted to cases where the friction is negligible (e.g. lubricated surfaces). It was shown in an earlier paper (Isaksson and Ståhle, 2001) that the effect of homogenous friction between the crack surfaces is a reduction of $K_{\mathrm{II}}$. At higher load of friction on the crack surfaces, opening of the crack is suppressed and mode II crack growth is promoted at crack growth. Thus, as long as the deflection of the crack propagation curve is small, the effect of friction on the crack extension is believed to be limited.

\section{Experimental analysis and results}

In the experiments reported, there is clear evidence that mode II cracks do initiate and propagate (cf. Reyes, 1991; Reyes and Einstein, 1991; Shen, 1993; Shen et al., 1995; Bobet and Einstein, 1998; Rao, 1999; or Bobet, 2000). The crack growth where reported to occur in a stable manner. The mode II crack growth initiates in all the experiments referred at the tip of straight pre-existing cracks and continues in the direction straight-ahead in $a$, on the macro level, slightly curved path. The researchers reported that observations on the surface of the specimens revealed that the cracks initiated at the tips of the pre-existing cracks as crushing of the material, or some material spalling from the surface, occurred. Phenomena that are characteristic of a compressive stress field regarding to the experimentalists referred. Furthermore, Bobet and Einstein (1998) reported that inspection of the mode II crack surfaces showed the presence of many small kink steps and crushed material. As our model, briefly described in Section 3, is based on an assumption of mode II crack growth, we conclude that the model may be suitable for circumstances as in the reported experiments.

For the experiments discussed, pictures on cracked samples are shown. In this investigation, these pictures were photocopied and enlarged and then, by simply measuring by hand, data points (coordinates) describing the mode II crack patterns could be obtained.

The crack patterns studied are not smooth. Sometimes the material in the samples was crushed, making it very hard to figure out the exact crack path, and sometimes it was even more difficult to realize where the pre-existing cracks ended and where the initiated mode II crack growth started. Thus care must be taken when evaluating the experiments, especially as crack contours on the microscale is considered. In some cases, when so-called material spalling has occurred, the measured data points were collected after the point where the crack growth continued after the spalling.

A Cartesian $(x-y)$ coordinate system is introduced with its origin coinciding with the pre-existing crack tip, as discussed in Section 3, and the resulting crack growth pattern emerged from the pre-existing cracks was analyzed. In Fig. 4a-f, traces of mode II crack propagation paths from the various experiments are 

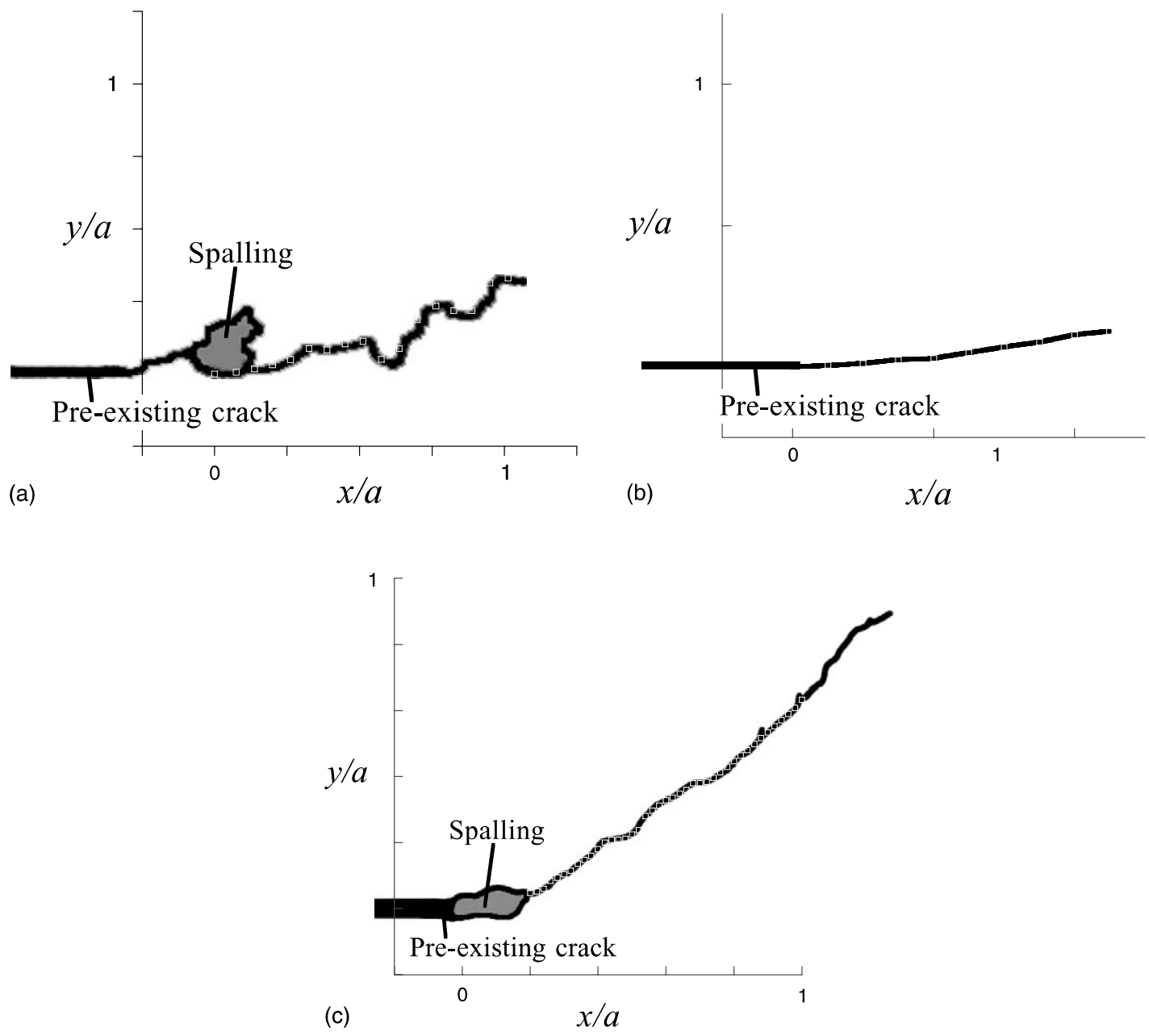

Fig. 4. Mode II crack patterns emerged from pre-existing cracks. The propagation paths are measured from various experiments reported in the literature. The $x$ - and $y$-axis are normalized with $a$, where $2 a$ is the length of the pre-existing cracks.

marked. The length of the internal pre-existing cracks is $2 a$ and the $x$-and $y$-axis in the plots are normalized with respect to $a$. The specimens were made of gypsum, except in the experiment reported by Rao (1999) where the pre-existing cracks were surface cracks in a specimen made of marble.

The collected coordinates were transferred to log-log diagrams, Fig. 5a-f, for each crack pattern measured. In that way, the exponent $b$ and the curvature parameter $\lambda$ in the assumed expression

$$
y=\lambda x^{b},
$$

could be determined by fitting a polynomial $\log y=\log \lambda+b \log x$ that fits the measured data in a leastsquare sense.

Sometimes, due to the above discussed spalling or pulverization of the material, the coordinates describing the crack growth is somewhat scattered. For this reason a few coordinates are neglected when fitting the polynomial. By inspection of Fig. 5a-f one may realize that this can be justified as it seems as the 


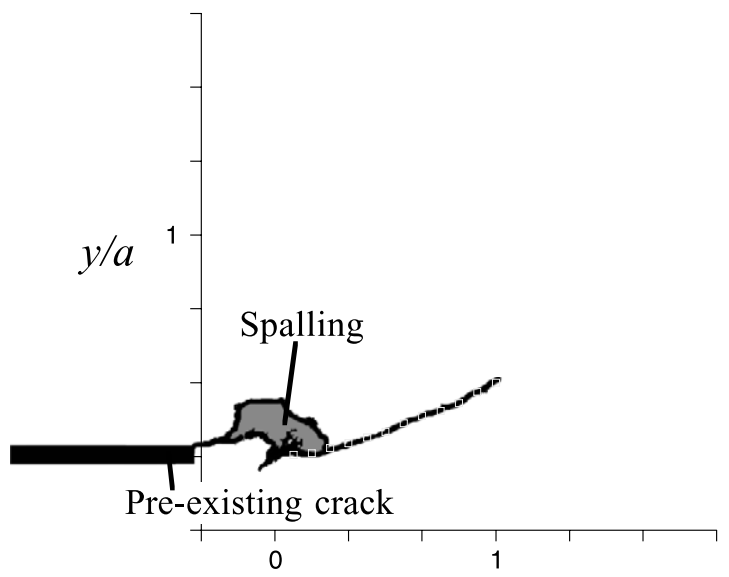

(d)

$$
x / a
$$

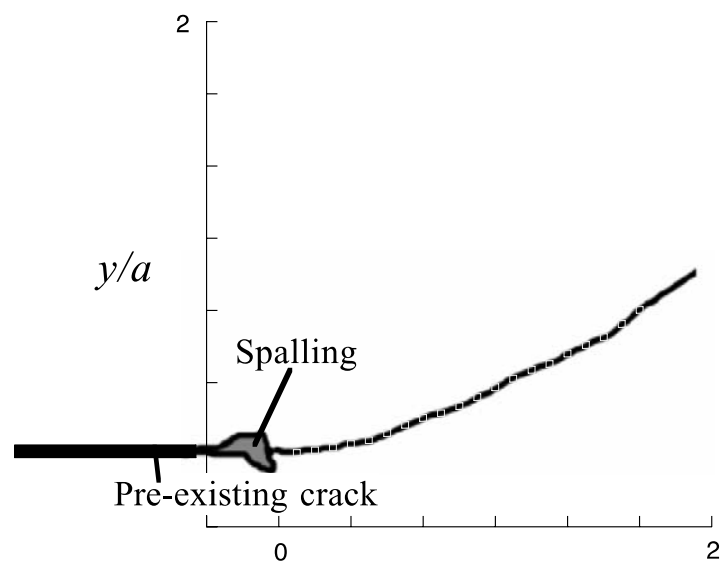

(e)

$x / a$

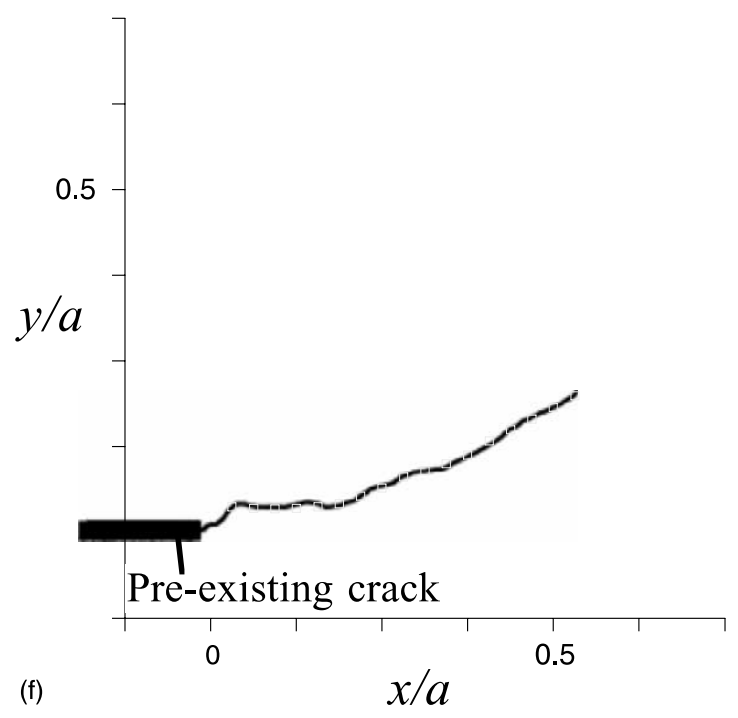

Fig. 4 (continued)

few coordinates removed do not follow the same trend as the others. Nevertheless they have great influence when finding the polynom in a least-square sense. All coordinates are marked in the diagrams and those, which are removed, are marked differently.

The results are summarized in Table 1 . The load angle $\theta$ and the theoretical value of $\lambda$ have been calculated according to the expression found in Appendix A. By examination of the results summarized in Table 1, it seems justified to conclude that the model can, on the macroscale, reproduce the mode II crack curvature obtained in the experiments. In all curves measured, the exponent $b$ was found to be in the interval [1.43-1.58], which support the crack path model analyzed as the analytical expression prescribes $b=3 / 2$ (i.e., $\gamma$ in Eq. (11)).

The results of the measured curvature parameter $\lambda$ also agree with the simplified model, but maybe not as good as the exponent $b$. This may be explained by the fact that the samples were crushed and pulverized 


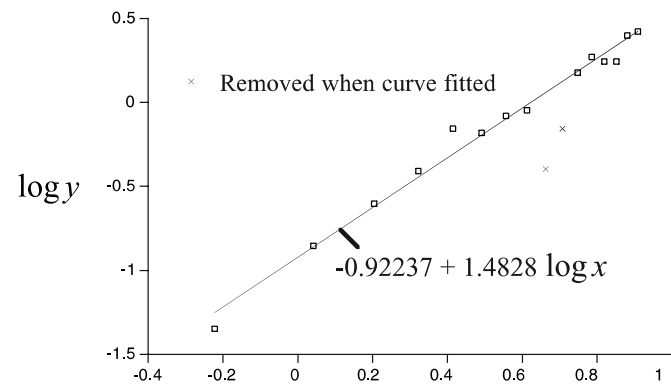

(a)

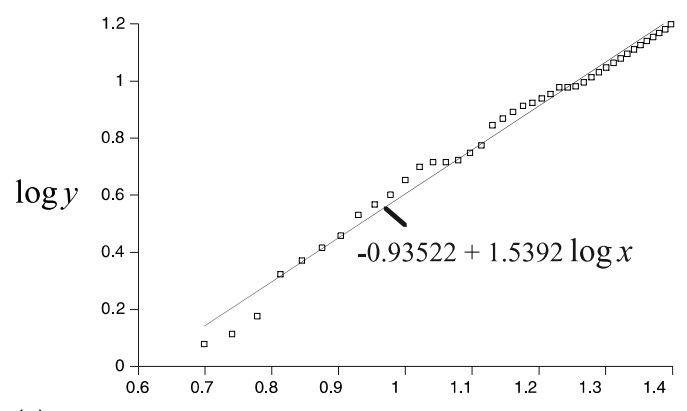

(c)



(e)

$\log x$
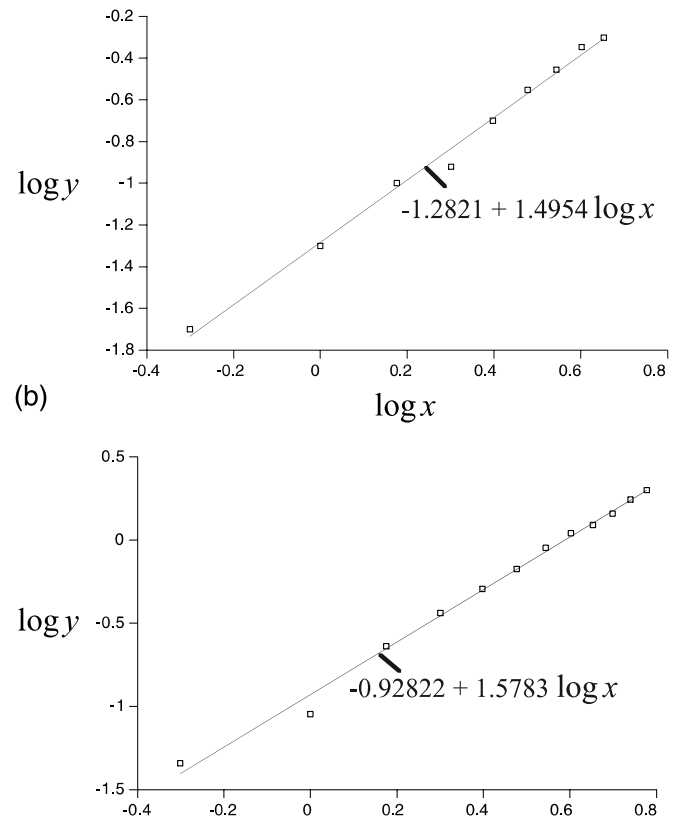

(d)

$\log x$

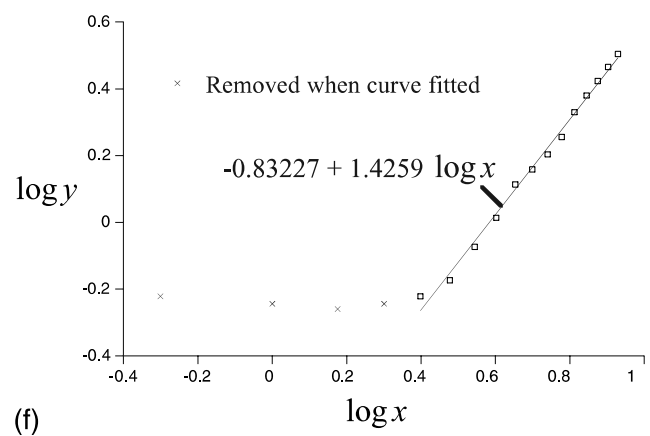

Fig. 5. Log-log diagram of the corresponding plots in Fig. 4a-f. The solid lines are least square fitted straight lines to the measured propagation paths.

Table 1

Results obtained for $\theta$ defined in Fig. 8, and $\lambda$ and $b$ given in Eq. (12)

\begin{tabular}{lllll}
\hline Experiment referred & Load angle $\theta\left(^{\circ}\right)$ & Theor. $\lambda$ & $\lambda$ & $b$ \\
\hline Fig. 11 in Bobet and Einstein (1998) & 60 & 0.133 & 0.120 & 1.48 \\
Fig. 6.8 in Rao (1999) & 50 & 0.049 & 0.052 & 1.49 \\
Fig. 2.7 in Reyes and Einstein (1991) & 30 & 0.133 & 0.116 & 1.54 \\
Fig. 3.23 in Reyes (1991) & 30 & 0.133 & 0.118 & 1.58 \\
Fig. 3.20 in Reyes (1991) & 30 & 0.133 & 0.116 & 1.53 \\
Fig. 6 in Shen et al. (1995) & 30 & 0.133 & 0.147 & 1.43 \\
\hline
\end{tabular}

during crack growth or that friction effects influenced. In most of the experiments spalling occurred in front of the pre-existing cracks wherefore the continued crack initiation and growth may also been affected by a 
disturbed stress field, as compared to the theoretical stress field far away used in the analytical model. Nevertheless, with all these assumptions in mind, $\lambda$ differs in general less than $15 \%$ as compared to the theoretical value predicted by the model (i.e., $c$ in Eq. (11)).

The experimental results lend confidence to the assumption of a macroscopic closed mode II crack propagation path described by Eq. (11). In fact, the agreement between the studied experiments and the propagation path prescribed by the model is astonishingly good considering the simplifying assumptions made for the analysis.

\subsection{A note on crack interaction}

In the experiments referred, as described in the Appendix A, two inclined pre-existing cracks were separated the distance $c$ and arranged parallel to each other with their respective crack plane separated the distance $s$. Bobet and Einstein (1998) report an interesting observation of the influence of ligament length $c$, i.e. the distance between the two pre-existing cracks. Up to a distance of $1 \frac{1}{2}$ times the crack length, i.e. $3 a$, the cracks are reported to affect each other during crack growth. As the two pre-existing cracks may influence each other stress fields at the crack tips, an investigation of the stress conditions under different load situations have been conducted. The numerical analysis was performed with the boundary element program FROCK (Chan et al., 1990; Bobet, 1997). FROCK is based on the displacement discontinuity method (DDM) and provides an easy way to model crack growth under various loads. The two inclined straight pre-existing cracks were modeled with 10 equally spaced special crack elements. The crack tips were modeled with square-root displacement discontinuity elements, while the body of the crack was modeled with linear displacement discontinuity elements. All the elements had the same length and the uniaxial load $P$ was applied at a distance far away from the cracks. The stresses in front of the inner tips of the cracks were calculated at varied inclination angles $\theta$ and ratio $s / c$, (the definitions of $\theta, s$ and $c$ as in the Appendix A). Note that the remote normal stresses $\sigma_{11}^{\infty}$ and $\sigma_{22}^{\infty}$ are compressive in front of the crack tips only for crack inclination angles $\theta$ ranging from $30^{\circ}$ to $60^{\circ}$, cf. Bobet and Einstein (1998). Calculations is performed for two cases of inclination angles $\theta$, (i.e., $\theta=45^{\circ}$ and $\theta=30^{\circ}$ ) and for each case, ligament lengths of $c=2 a$ and $c=4 a$ are compared. In all the computed cases $s=1 / 2 a$.

Fig. 6a-d show the calculated stresses $\sigma_{11}, \sigma_{22}$ and $\sigma_{12}$ along the crack plane at distance $x / a$ in front of the inner tips. The stresses are normalized with the load $P$. As can be seen the calculated compressive normal stresses $\sigma_{11}$ and $\sigma_{22}$ are somewhat different due to the interaction between the crack tips for $c=2 a$ than for $c=4 a$. At $c=4 a$ the stress field in front of the inner crack tip is approximate at the same magnitude as compared to the stress field from an isolated crack tip, Fig. 6e and $\mathrm{f}$, whereas the stresses $\sigma_{11}$ and $\sigma_{22}$ are more influenced by the interacting tip when $c=2 a$. Thus, the ligament length $c$ has an influence on the normal stress difference term $\sigma_{11}-\sigma_{22}$ in the plane of the pre-existing cracks. The shear stress $\sigma_{12}$, however, remains approximately at the same stress magnitude, independent of $c$ in the computations.

Fig. 7 indicates that the normal stress difference $\sigma_{11}-\sigma_{22}$ in front of the inner tip is much more sensitive to the ligament length $c$ at $\theta=45^{\circ}$ then at $\theta=30^{\circ}$. When $\theta=45^{\circ}$ and $c=2 a$, the normal stress difference $\sigma_{11}-\sigma_{22}$ in front of the crack may become around 9.5 times as high as compared to when $c=4 a$. The corresponding highest ratio becomes 1.4 for $\theta=30^{\circ}$, As discussed, the normal stress difference $\sigma_{11}-\sigma_{22}$ may have a strong effect on the deflection of the crack extension, according to the expressions in Eqs. (3), (10) and (11). The conclusion is that, according to the simplified model, the influence from an interacting crack tip, on the crack deflection, is more pronounced when the cracks are inclined $\theta=45^{\circ}$ than when $\theta=30^{\circ}$. This may explain why the mode II cracks obtained in the experiments, cf. Bobet and Einstein (1998), were reported to affect each other during crack growth for ligament length $c \leqslant 3 a$.

However, the interacting effect from the other crack tip may be limited in the load cases investigated here since the calculated ratio $\left.\left(\sigma_{11}-\sigma_{22}\right)\right|_{c=2 a} /\left.\left(\sigma_{11}-\sigma_{22}\right)\right|_{c=4 a}$ in front of the crack tip appears to be low for 

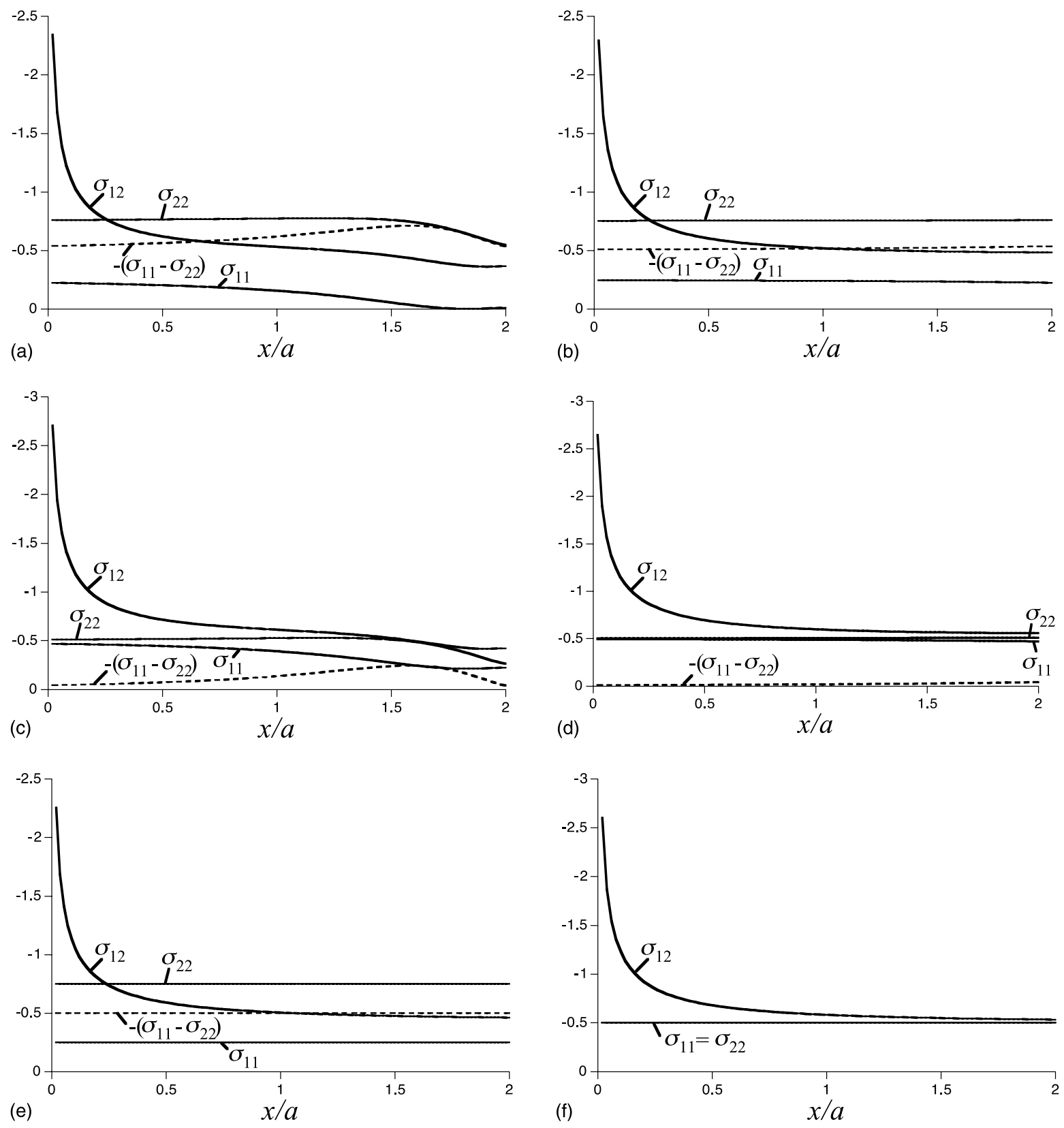

Fig. 6. Normalized in-plane stresses in front of the inner tips of the pre-existing cracks of length $2 a$. (a) Inclination angle $\theta=30^{\circ}$ and $c=2 a$, (b) inclination angle $\theta=30^{\circ}$ and $c=4 a$, (c) inclination angle $\theta=45^{\circ}$ and $c=2 a$, (d) inclination angle $\theta=45^{\circ}$ and $c=4 a$, (e) an isolated crack with inclination angle $\theta=30^{\circ}$ and (f) an isolated crack with inclination angle $\theta=45^{\circ}$.

$\theta=30^{\circ}$ (and $\theta=60^{\circ}$ ), Fig. 7. Nevertheless, even though it may be small, the interaction effect can be one of the possible reasons for the mismatch between the calculated and measured $\lambda$ in Table 1. 


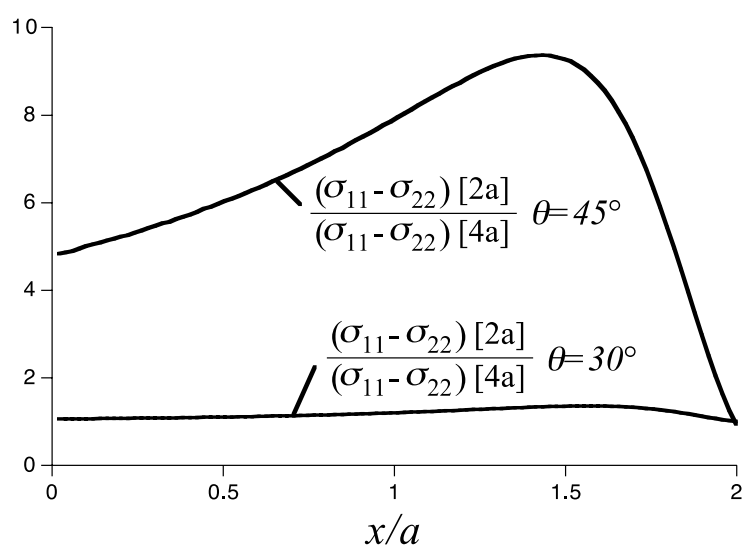

Fig. 7. The ratio between the in-plane normal stress difference term $\sigma_{11}-\sigma_{22}$ at $c=2 a$ and at $c=4 a$ for crack inclinations $\theta=30^{\circ}$ and $\theta=45^{\circ}$.

\section{Conclusions}

A number of comparisons between experiments showing the traces of mode II crack growth under compressive loading reported in literature and the curvature prescribed by the analytical model discussed by the authors elsewhere (Isaksson and Ståhle, 2001) show good agreements. The simplified model is based on an assumption of crack growth when the crack is constrained to remain closed due to high compressive stresses. The experiments referred have been conducted under uniaxial and biaxial compressive load and demonstrably cracked in mode II in samples made of brittle materials. By analyzing and evaluate the experimental mode II crack growth paths emerged from pre-existing cracks, the conclusion is that the model can not describe the more realistic microscopic failure in detail. Since mode II cracks on the microscale depend on inhomogenities in the material the closed crack paths observed are not smooth. Nevertheless, the analyzed model shows qualitatively a very good agreement with the reported experimental observations of the curvature of closed macroscopic mode II cracks subjected to overall compression.

The main conclusions obtained are:

- Crack patterns experimentally observed seems to indicate that the path of mode II cracks under compression in some brittle materials follow a crack propagation path described by $y=\lambda x^{b}$. This is strongly supported by the measured values obtained. In fact, the agreement between the experiments and the propagation path prescribed by the model, in which $b=3 / 2$, is astonishingly good considering the simplifying assumptions made for the analysis. In the studied experiments, $b$ was found in the interval [1.431.58].

- An investigation of the curvature parameter $\lambda$ has also been performed. The analytical model prescribes for a curved crack growth $\lambda=-8 / 39(2 \pi)^{1 / 2}\left[\sigma_{11}^{\infty}-\sigma_{22}^{\infty}\right] / K_{\text {II }}$, where $K_{\text {II }}$ is the mode II stress intensity factor of the parent crack, $\sigma_{11}^{\infty}$ is the remote compressive normal stress parallel with the crack plane and $\sigma_{22}^{\infty}$ is the remote compressive normal stress perpendicular to the crack plane. The conclusion is that $\lambda$ also agree very good with the simplified model, even though not as good as the exponent $b$. The measured $\lambda$ differs in general less than $15 \%$ as compared to the theoretical values predicted by the model, which is considered to be surprisingly good.

- The influence of the ligament length between two parallel cracks, on the crack deflection, has been investigated by using a boundary element program. The conclusion is that the influence from the stress 
field of an interacting crack tip, on the crack deflection, is more pronounced when the cracks are inclined $\theta=45^{\circ}$ than when $\theta=30^{\circ}$ according to the simplified model. Even though it may be small, the interaction effect can be a reason for the mismatch between the measured $\lambda$ and the curvature prescribed by the model.

\section{Acknowledgements}

Financial support from the Swedish Research Council for Engineering Sciences (TFR) is gratefully acknowledged. The authors are indebted to Professor H.H. Einstein at MIT and the developers Dr. H.C.M. Chan and Professor A. Bobet for providing source code of the boundary element program FROCK.

\section{Appendix A. Description of the referred experiments}

In (Reyes, 1991; Reyes and Einstein, 1991; Shen et al., 1995; and Bobet and Einstein, 1998) samples in a brittle rock-like material, gypsum, were designed in the following way:

Two pre-existing cracks of length $2 a$ were arranged in the central part of specimens as described by Fig. 8. The length of the ligament between the inner tips was $c$. Different inclinations $\theta$ of the fractures and the ligament (i.e. the ratio $s / c$ ) were then used to investigate the influence of the fracture geometry on failure modes. The samples were loaded uniaxially or biaxially while the fracture initiation and propagation process was monitored with a video camera.

The length of the pre-existing cracks were $2 a=12.7 \mathrm{~mm}$ while the samples were manufactured in the dimension $24 a \times 12 a$ (height $\times$ width). Since the length of the fractures was much smaller than the thickness of the specimen $(30 \mathrm{~mm})$, the fractures can be considered to be in plane strain conditions.

The inclination of the two pre-existing cracks in the experiments, $\theta$, varied from $30^{\circ}$ to $60^{\circ}$ in steps of $15^{\circ}$. However, only when the ligament inclination ratio $s / c<1 / 3$, the specimens where reported to fracture in

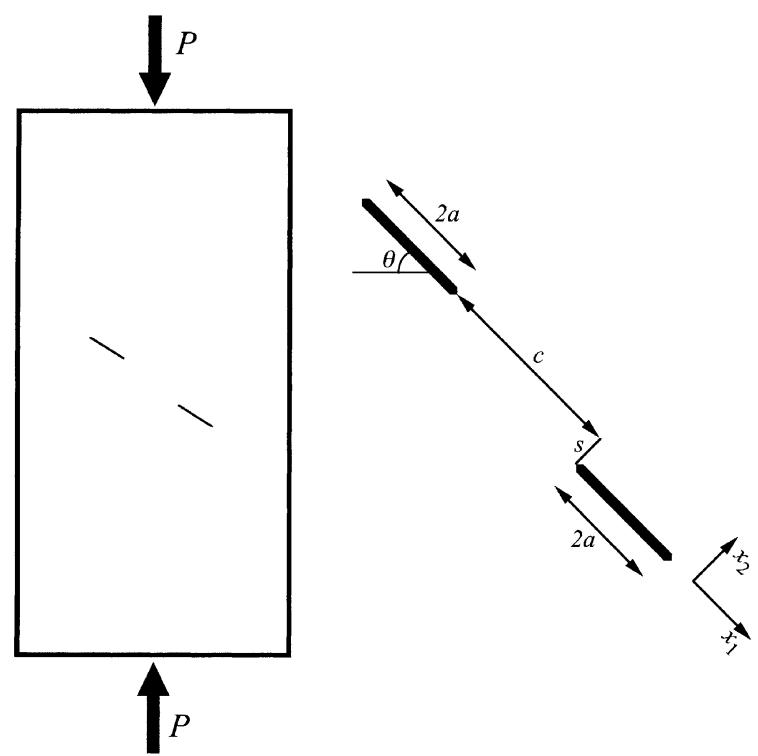

Fig. 8. Experimental setup and definitions of quantities. 
pure mode II. At larger bridge inclinations the specimens fractured in mode I or a combination of mode I and mode II. Only the crack paths achieved under shear mode are discussed in this investigation.

By the definitions in the Fig. 8 may the uniaxial load $P$ of the specimens be transferred to a local Cartesian $\left(x_{1}, x_{2}\right)$ coordinate system. The far field stresses may be expressed as:

$$
\begin{aligned}
& \sigma_{11}=-P \sin ^{2} \theta, \\
& \sigma_{22}=-P \cos ^{2} \theta,
\end{aligned}
$$

and

$$
\sigma_{12}=-P \sin \theta \cos \theta
$$

If the geometry of the specimens is regarded as infinite sized cracked plates, the mode II stress intensity factor $K_{\mathrm{II}}$ at the crack tips may be expressed as:

$$
K_{\mathrm{II}}=\sigma_{12} \sqrt{\pi a},
$$

where $\sigma_{12}$ is the uniform in-plane shear stress at infinity. If friction is neglected on the crack surfaces and crack interaction is disregarded, $\sigma_{12}$ is obtained from Eq. (A.3).

An approximate theoretical expression of the curvature parameter $\lambda$ in Eq. (12) can be obtained by use of Eqs. (A.1)-(A.4):

$$
\lambda=-\frac{8 \sqrt{2 \pi}}{39} \frac{1}{\sqrt{\pi a}} \frac{\sin ^{2} \theta-\cos ^{2} \theta}{\sin \theta \cos \theta} .
$$

As can be seen in Eq. (A.5), $|\lambda|$ is symmetric around the inclination angle $\theta=45^{\circ}$, wherefore $|\lambda|$ at $\theta=30^{\circ}$ is equal to $|\lambda|$ at $\theta=60^{\circ}$.

In the experiments reported by Rao (1999) the laboratory setup is different. Here, mode II cracks were examined in square double-notched specimens in a so-called shear-box. Two inclined straight surface cracks are considered, each of length $a$. The ligament length is $W-2 a$, where $W$ is the width of the specimen. A simplified model for calculating the ratio $\left(\sigma_{11}-\sigma_{22}\right) / \sigma_{12}$ may under these circumstances be written as

$$
\left(\sigma_{11}-\sigma_{22}\right) / \sigma_{12}=\frac{\sin ^{2} \theta-\cos ^{2} \theta}{\sin \theta \cos \theta} \frac{1}{1-2 a / W},
$$

where the term $1 /(1-2 a / W)$ come from an equilibrium condition of the nominal stress in the ligament computed from the ratio load/area, an elementary formula which does not take account of the stress concentration "peak" at the crack tip. As the crack considered is a surface crack, the curvature parameter $\lambda$ is in this case approximated by the simplified expression

$$
\lambda=-\frac{8 \sqrt{2 \pi}}{39} \frac{1}{1.12 \sqrt{\pi a}} \frac{\sin ^{2} \theta-\cos ^{2} \theta}{\sin \theta \cos \theta} \frac{1}{1-2 a / W} .
$$

\section{References}

Bilby, B., Eshelby, J.D., 1968. Dislocations and the theory of fracture. In: Liebowitz, H. (Ed.), Fracture and Advanced Treatise, vol. 1. Academic Press, New York, pp. 99-182.

Bobet, A., 1997. Fracture coalescence in rock materials: experimental observations and numerical predictions. Ph.D. thesis. Massachusetts Institute of Technology. Cambridge, MA, USA.

Bobet, A., Einstein, H.H., 1998. Fracture coalescence in rock-type materials under uniaxial and biaxial compression. Int. J. Rock Mech. Min. Sci. 35, 863-888. 
Bobet, A., 2000. The initiation of secondary cracks in compression. Eng. Fract. Mech. 66, 187-219.

Broberg, K.B., 1987. On crack paths. Eng. Fract. Mech. 28, 663-679.

Broberg, K.B., 1999. Cracks and Fracture. Academic Press, London, UK.

Chan, H.C.M., Li, V., Einstein, H.H., 1990. A hybridized displacement discontinuity and indirect boundary element method to model fracture propagation. Int. J. Fract. 45, 263-282.

Cotterell, B., Rice, J.R., 1980. Slightly curved or kinked cracks. Int. J. Fract. 16, 155-169.

Erdogan, F., Sih, G.C., 1963. On the crack extension in plates under plane loading and transverse shear. J. Basic Eng. 85, 519-527.

He, M.Y., Hutchinson, J.W., 1989. Kinking of a crack out of an interface. J. Appl. Mech. 56, 270-278.

Hayashi, K., Nemat-Nasser, S., 1981a. On branched, interface cracks. ASME J. Appl. Mech. 48, 529-533.

Hayashi, K., Nemat-Nasser, S., 1981b. Energy-release rate and crack kinking under combined loading. J. Appl. Mech. 48, 520-524.

Isaksson, P., Ståhle, P., 2001. Crack kinking under high pressure in an elastic-plastic material. Int. J. Fract. 108, 351-366.

Isaksson, P., Ståhle, P., 2001. Prediction of shear crack growth direction under compressive loading and plane strain conditions. Int. J. Fract., in press.

Jung, S.J., Enbaya, M., Whyatt, J.K., 1992. The study of fracture of brittle rock under pure shear loading. In: Mybe, L.R., Tsang, C.F., Cook, N.G.W., Goodman, R.E. (Eds.), Fractured and Jointed Rock Masses: Proceedings of the Conference on fractured and jointed rock masses. Lake Tahoe, CA, USA, pp. 457-463.

Lajtai, E., 1974. Brittle fracture in compression. Int. J. Fract. 10, 525-536.

Leblond, J.-B., Frelat, J., 2000. Crack kinking from an initially closed crack. Int. J. Solids Struct. 37, $1595-1614$.

Li, S., 1999. Modeling fracture and deformation of brittle rock under compressive loading. Ph.D. thesis. The University of Manitoba, Winnipeg, Canada.

Lo, K.K., 1978. Analysis of branched cracks. J. Appl. Mech. 45, 797-802.

Melin, S., 1986. When does a crack grow under mode II conditions? Int. J. Fract. 30, 103-114.

Melin, S., 1987. Fracture from a straight crack subjected to mixed mode loading. Int. J. Fract. 32, $257-263$.

Melin, S., 1989. Why are crack paths in concrete and mortar different from those in PMMA? Mater. Struct. $22,23-27$.

Petit, J.-P., Barquins, M., 1988. Can natural faults propagate under mode II conditions? Tectonics 7, $1243-1256$.

Rao, Q., 1999. Pure shear fracture of brittle rock - a theoretical and laboratory study. Ph.D. thesis. Luleå University of Technology, Sweden.

Reyes, O., 1991. Experimental study and analytical modelling of compressive fracture in brittle materials. Ph.D. thesis. Massachusettes Institute of Technology, MA, USA.

Reyes, O., Einstein, H.H., 1991. Failure mechanisms of fractured rock-a fracture coalescence model. In: Wittke, W. (Ed.), Proceedings of the International Congress on Rock Mechanics. Aachen, Germany, pp. 333-340.

Shen, B., 1993. Mechanics of fractures and intervening bridges in hard rocks. Ph.D. thesis. Royal Institute of Technology, Stockholm, Sweden.

Shen, B., Stephansson, O., Einstein, H.H., Ghahreman, B., 1995. Coalescence of fractures under shear stresses in experiments. J. Geophys. Res. 100, 5975-5990.

Suresh, S., 1994. Fatigue of Materials. University Press, Cambridge, UK. 